\title{
Multiple-Model Optimization of Proportional Integral Controllers on Canals
}

\author{
P. J. van Overloop ${ }^{1}$; J. Schuurmans ${ }^{2}$; R. Brouwer ${ }^{3}$; and C. M. Burt, M.ASCE ${ }^{4}$
}

\begin{abstract}
Canals or open channels that convey water often consist of pools in series separated by control structures. Successful implementation of water-level control with these structures using decentralized proportional integral (PI) controllers depends heavily on the tuning of the control parameters. These parameters are hard to determine due to the interactions between the pools and the varying flow conditions in the canal. This paper presents a procedure for tuning any linear controller (including decentralized PI controllers) that guarantees stability of the controlled canal. It minimizes a cost function that weights the water-level deviations from the target level against control efforts at both low- and high-flow conditions. The procedure is tested on a model of the Umatilla Stanfield Branch Furnish Canal in Oregon. The tests show the capability of the procedure to deal with the pool interactions. The results of a realistic turnout schedule applied to the controlled canal show the high performance of the controllers (small water-level deviations in all pools) over varying flow conditions.
\end{abstract}

CE Database subject headings: Optimization models; Canals; Open channels; Control structures; Hydraulic structures.

\section{Introduction}

Canals or open channels that convey water often consist of pools in series separated by control structures. Most automated canals have hydraulic check structures or simple proportional electromechanical controllers. Although there is ample literature regarding the use of proportional integral (PI) algorithms for canal control, there are very few successful field implementations in irrigation districts. One of the reasons for the slow adoption of PI algorithms for canal control is the lack of tools for tuning the algorithm parameters. Finding these parameters can be a difficult and time-consuming problem for mainly two reasons. First, control actions of one PI controller disturb the water level in an adjacent pool. If this disturbing effect is not taken into account, disturbance amplification is likely to happen. Second, the dynamics of open-channel flow are highly nonlinear. Therefore, tuning the controllers for one flow condition does not guarantee satisfactory control in another flow condition. These two problems are not unique to PI control; they apply to any control algorithm, be it linear quadratic Gaussian, model predictive control, etc.

${ }^{1} \mathrm{PhD}$ Researcher, Delft Univ. of Technology, Section Water Management, Stevinweg 1, 2628CN, Delft, The Netherlands.

${ }^{2}$ Control Specialist, Canal Control, Overakker 27, 4614GZ, Bergen op Zoom, The Netherlands.

${ }^{3}$ Professor, Delft Univ. of Technology, Section Water Management, Stevinweg 1, 2628CN, Delft, The Netherlands.

${ }^{4}$ Professor and Director, Irrigation Training and Research Center, BioResources and Agricultural Engineering Dept., California Polytechnic State Univ., San Luis Obispo, CA 93407.
This paper presents a procedure for tuning any linear controller (including decentralized PI controllers) that guarantees stability of the control loops. It minimizes a cost function that weights the water-level deviations from the target levels against the control efforts. The tuning procedure makes use of a set of linear models (each valid for small variations around one flow condition).

\section{Decentralized versus Centralized Control}

Decentralized control (sometimes called "local control" or "distributed control") is where the control actions are computed using only measurements taken near the structure. With centralized control, measurements from all sites can be used to compute the

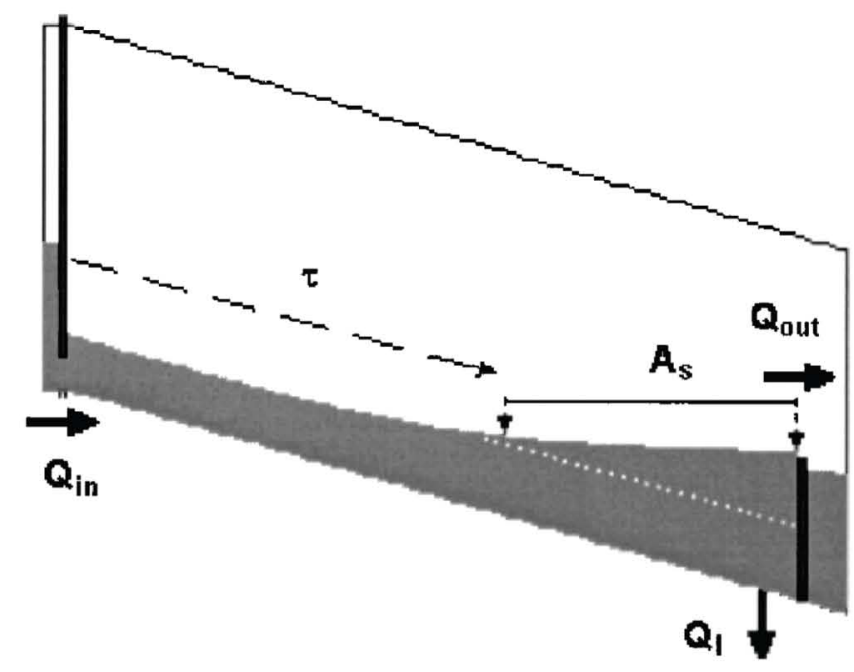

Fig. 1. Characterization of a pool with integrator delay model 


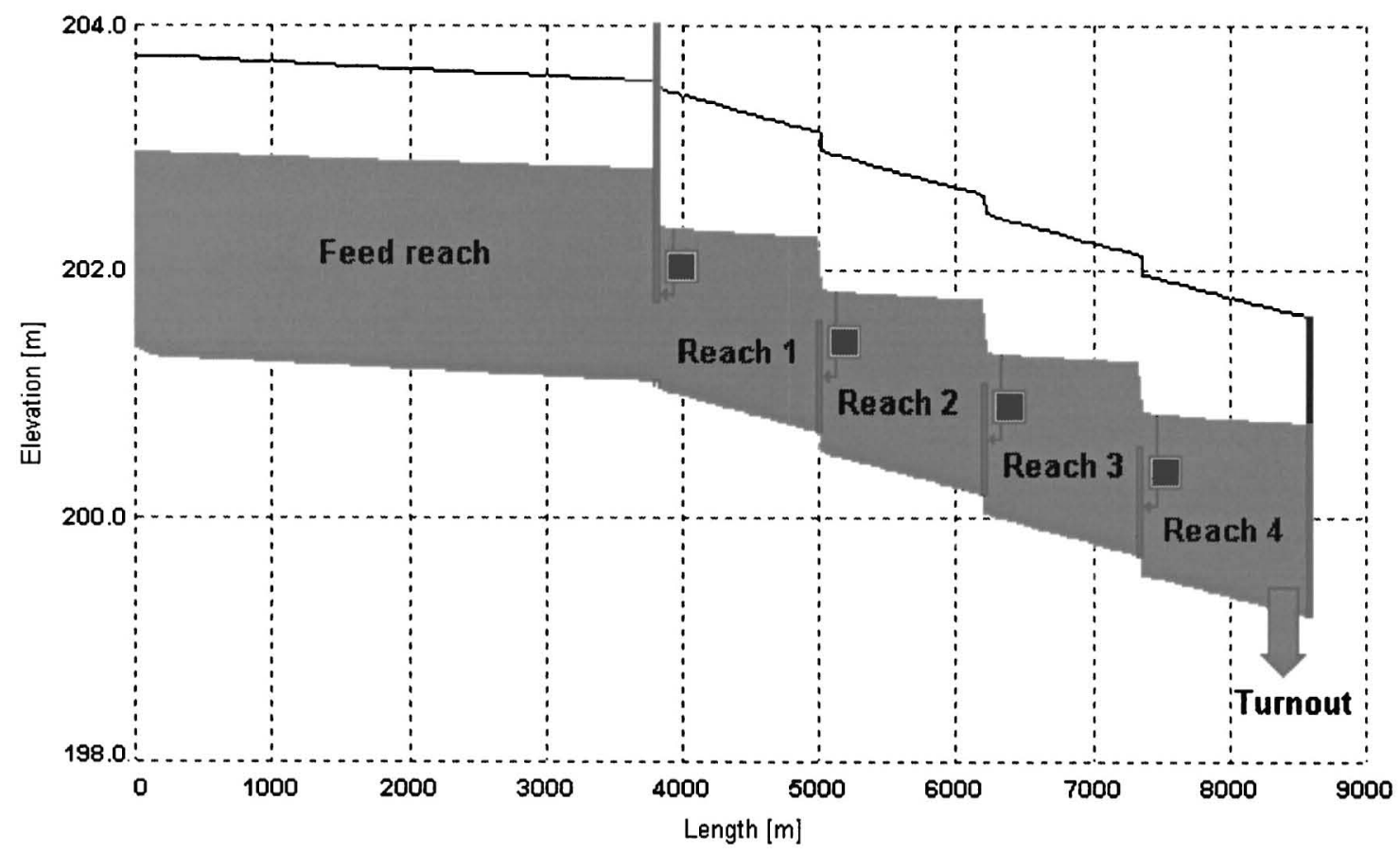

Fig. 2. Model of Umatilla Stanfield Branch Furnish Canal: Decentralized control with the target level immediately downstream of each check structure

control actions for all structures along the canal. As more information can be used, centralized control can potentially result in a higher performance than decentralized control (Schuurmans et al. 1999; Wahlin and Clemmens 2002). On the other hand, centralized control requires more hardware, which makes the chance of system failure higher. For example, communication links can easily be damaged by cable cuts or radio interference.

Under one scenario both centralized and decentralized control might be available. During normal operating conditions, centralized control is active and water levels are maintained close to their target levels, even under largely fluctuating turnout flows. When there is a failure, the system can be switched to decentralized control until the problem is solved. During this period the turnout flows should not be allowed to change (at least not too much), as the ability to deal with disturbances is lower for decentralized control than for centralized control (Overloop et al. 2001).

\section{Initial Controller Tuning}

A difficulty in controller design is that the controlled pools in series disturb one another. When decentralized PI control is

Table 1. Controller Parameters for the Umatilla Stanfield Branch Furnish Canal Resulting from Tuning Individual Pools without Considering Adjacent Pool Interactions

\begin{tabular}{lccc}
\hline Pool & $K_{p}$ & $K_{i}$ & $F_{c}$ \\
\hline 1 & -16.56 & -0.750 & 0.762 \\
2 & -16.31 & -0.718 & 0.769 \\
3 & -16.84 & -0.624 & 0.777 \\
4 & -16.31 & -0.717 & 0.768 \\
\hline
\end{tabular}

applied, disturbance amplification can occur. This happens when the PI controller in a pool is designed to have high performance on that pool, without considering the effect it has on the other pools. Here, high performance is defined as

- Good disturbance rejection (small fluctuations of the controlled variables under varying turnout flows); and

- Smooth control actions of the gate positions.

In this paper a variation of the PI controller is considered, called the PIF control. The "F" is added to indicate that a firstorder filter is applied in series with the PI controller. This filter is added when resonance waves (surge waves) play a dominant role in the water movements. These oscillations are common in short or flat pools, especially at low flow. The filter algorithm used is

$$
\Delta Q_{(k)}=K_{p} \cdot\left[e_{f_{(k)}}-e_{f_{(k-1)}}\right]+K_{i} \cdot e_{f_{(k)}}
$$

where $\Delta Q=$ required flow change through control structure in cubic meters per second; $k=$ time index; $K_{p}=$ proportional gain; $K_{i}=$ integral gain; $e_{f_{(k)}}=$ filtered error at present control time step in meters; $e_{f_{(k-1)}}=$ filtered error at previous control time step in meters, $T_{c}$ seconds ago; and $T_{c}=$ control time step in seconds.

The filtered water level error, $e_{f}$, is computed by

Table 2. Controller Parameters Resulting from Multiple-Model Optimization

\begin{tabular}{lccc}
\hline Pool & $K_{p}$ & $K_{i}$ & $F_{c}$ \\
\hline 1 & -16.56 & -1.006 & 0.762 \\
2 & -16.31 & -0.479 & 0.768 \\
3 & -16.84 & -0.356 & 0.777 \\
4 & -16.31 & -0.288 & 0.768 \\
\hline
\end{tabular}




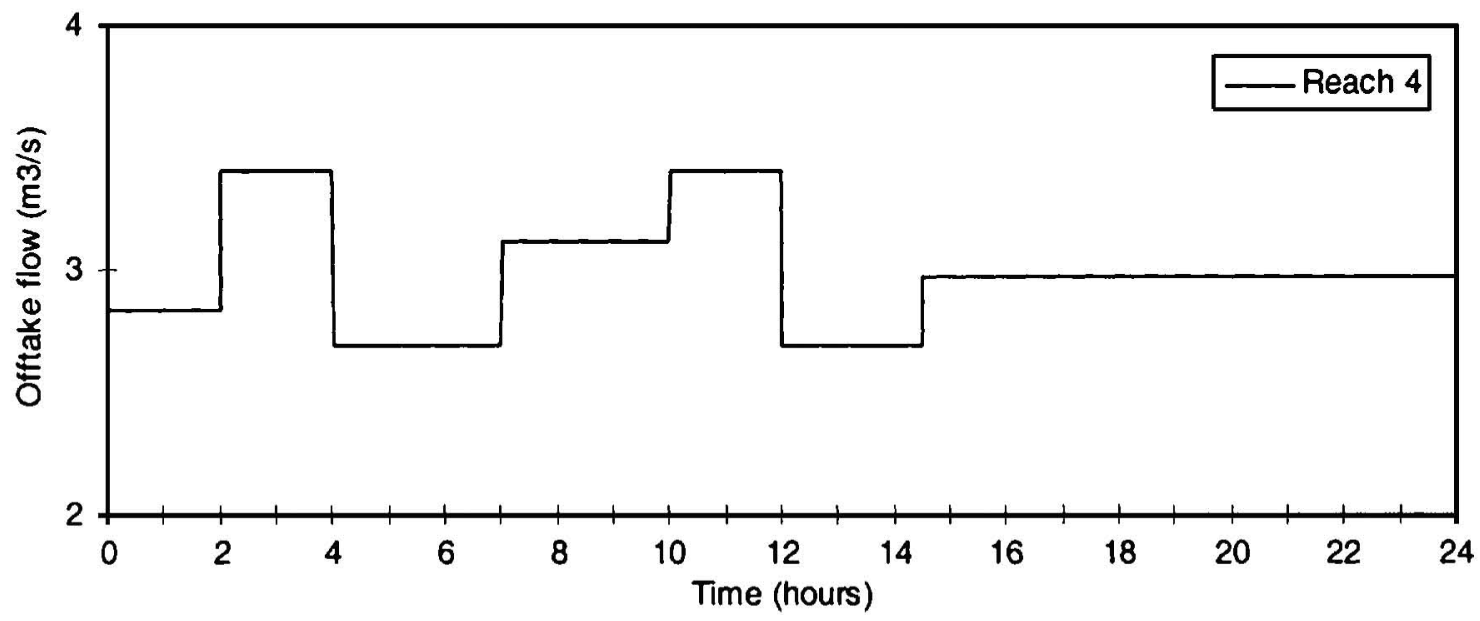

Fig. 3. Realistic turnout (offtake) schedule used at last turnout in Umatilla Stanfield Branch Furnish Canal

$$
e_{f_{(k)}}=F_{c} \cdot e_{f_{(k-1)}}+\left(1-F_{c}\right) \cdot e_{(k)}
$$

where $F_{c}=$ filter constant; $e=$ error or deviation (water level minus target level) in meters.

PIF controllers result in the flow changes that must be applied at the control structures. This flow change is translated into the gate position change by inverting the control structure's discharge equation.

In earlier research (Schuurmans 1997) tuning rules have been derived for resonance-dominated pools that result in high performance in each pool separately:

$$
\begin{gathered}
K_{p}=\frac{1}{2} \cdot \sqrt{\frac{A_{s, \text { mean }} \cdot \omega_{r}}{R_{p}}} \\
K_{i}=\frac{T_{c} \cdot \omega_{r}}{12 \cdot R_{p}}
\end{gathered}
$$

$$
F_{c}=e^{-\left(T_{c} / \sqrt{\left.\left(A_{s, \text { mean }} \cdot R_{p}\right) / \omega_{r}\right)}\right.}
$$

where $A_{S \text {, mean }}=$ average storage area over all operating conditions in square meters; $R_{p}=$ resonance peak at low flow; and $\omega_{r}$ $=$ frequency of resonance peak at low flow in radians per second.

\section{Multiple-Model Optimization of Control Parameters}

Canal dynamics can be described by the Saint-Venant equations. These equations are nonlinear, partial differential equations with no closed-form solution. Such equations are difficult to handle in control design and tuning. A method often used to overcome this difficulty is to discretize the partial differential equations and linearize them, which results in a model consisting of ordinary linear differential equations.

The linear models used in this paper are known as the integrator delay (ID) models (Schuurmans 1997). These models charac-
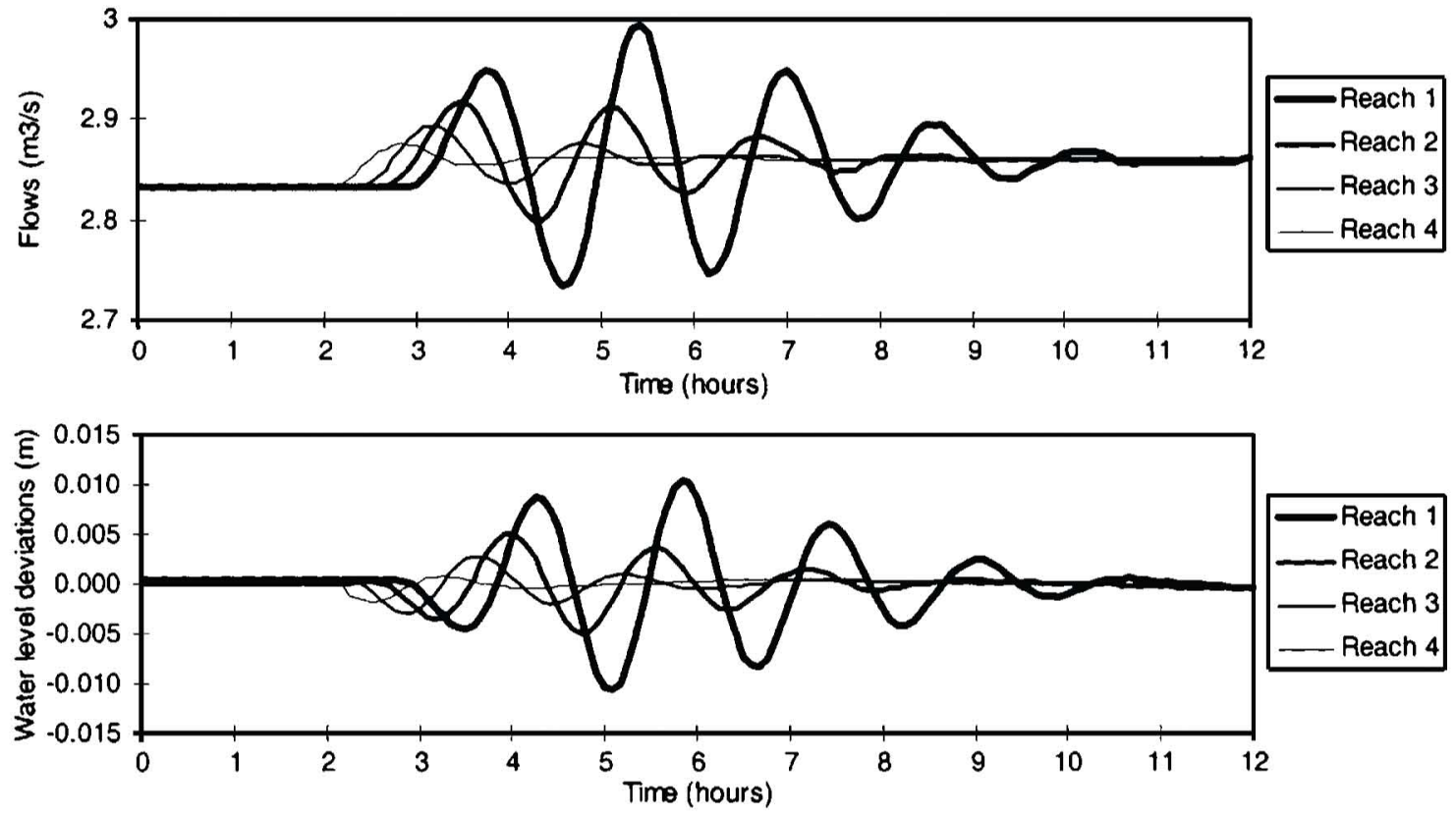

Fig. 4. Independent tuning of Umatilla Stanfield Branch Furnish Canal controllers for high performance in each pool 

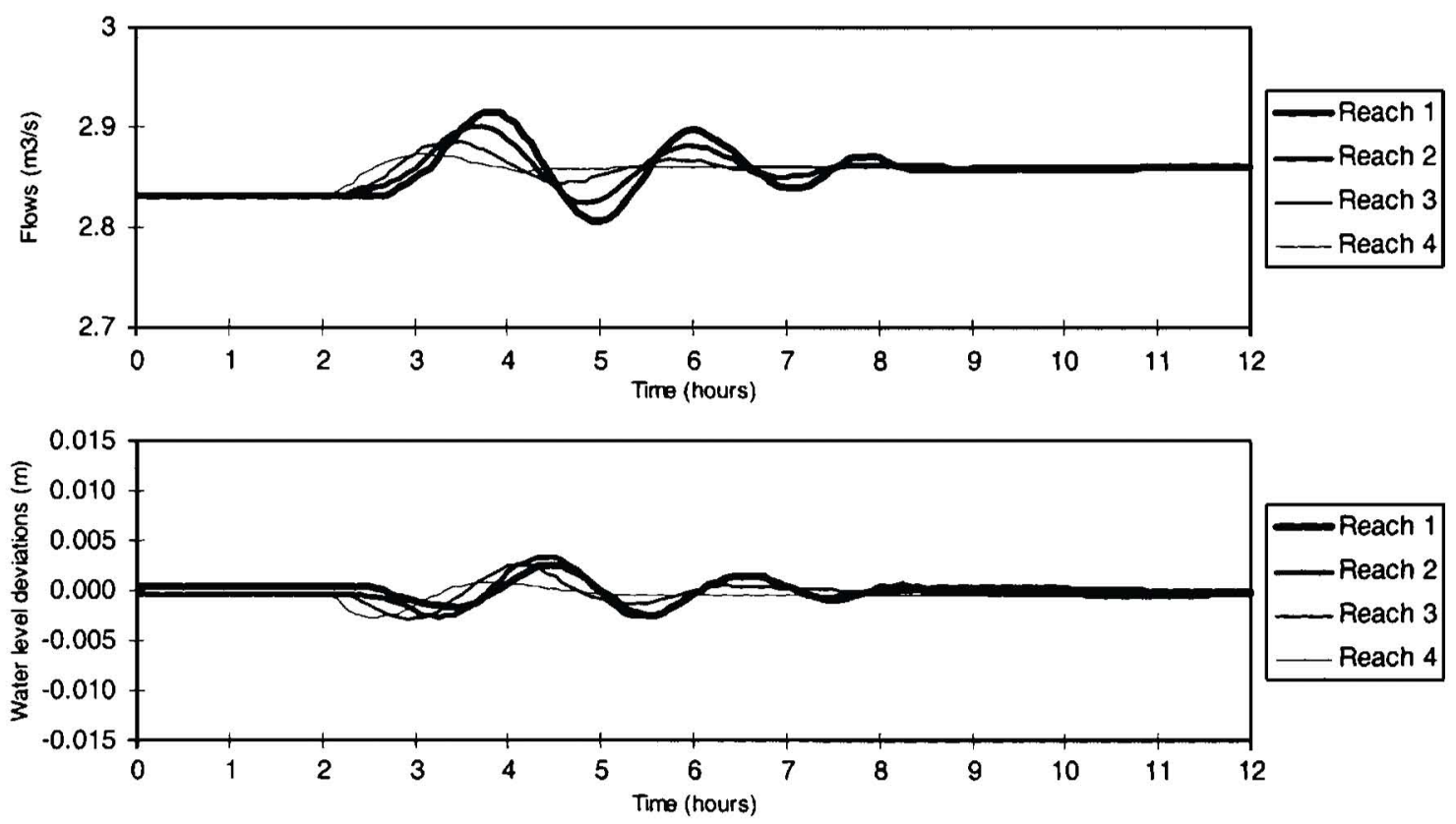

Fig. 5. Simulation of Umatilla Stanfield Branch Furnish Canal using optimized controller parameters

terize the dynamic behavior of the canal through the storage areas in the backwater section $A_{s}$ and delay times $\tau$ of the pools. $A_{s}$ corresponds to the "integrator" part of the ID model, while $\tau$ corresponds to the "delay" part. Each pool is described by the following differential equation:

$$
\frac{d h(t)}{d t}=\frac{Q_{\text {in }}(t-\tau)-Q_{\text {out }}(t)-Q_{1}(t)}{A_{s}}
$$

where $h=$ water level at the measurement location in meters; $t$ $=$ time in seconds; $Q_{\text {in }}=$ flow through upstream structure in cubic meters per second; $Q_{\text {out }}=$ flow through downstream structure in cubic meters per second; $Q_{1}=$ flow through turnout (offtake) structure in cubic meters per second; $\tau=$ delay time from control structure to measurement location in seconds; and $A_{s}=$ storage area of backwater part in square meters. Fig. 1 shows a longitudinal profile of one pool and the way this pool is characterized by $\tau$ and $A_{s}$.
Such a model can be a good approximation of the SaintVenant equations, as long as the flow rates and water levels do not change too much (i.e., a few percentages). There is a vast amount of knowledge on the control of such systems. However, a controller that is tuned using such a model may be unstable when applied to the real system, since flow rates vary considerably in practice. A more reasonable and useful simplification of the Saint-Venant equations is to model them as a set of linear models. A set that is very advantageous in tuning and easy to use is the multiple-model set. In this set, the pool is described by one linear model for each set of flows.

For this set the multiple-model optimization technique has been proposed (Geromel and Bernussou 1979). In this technique, a linear controller is tuned in such a way that it stabilizes all models (for all sets of flows) and optimizes an objective function that is a sum of individual objective functions, each valid for one of the models from the set.

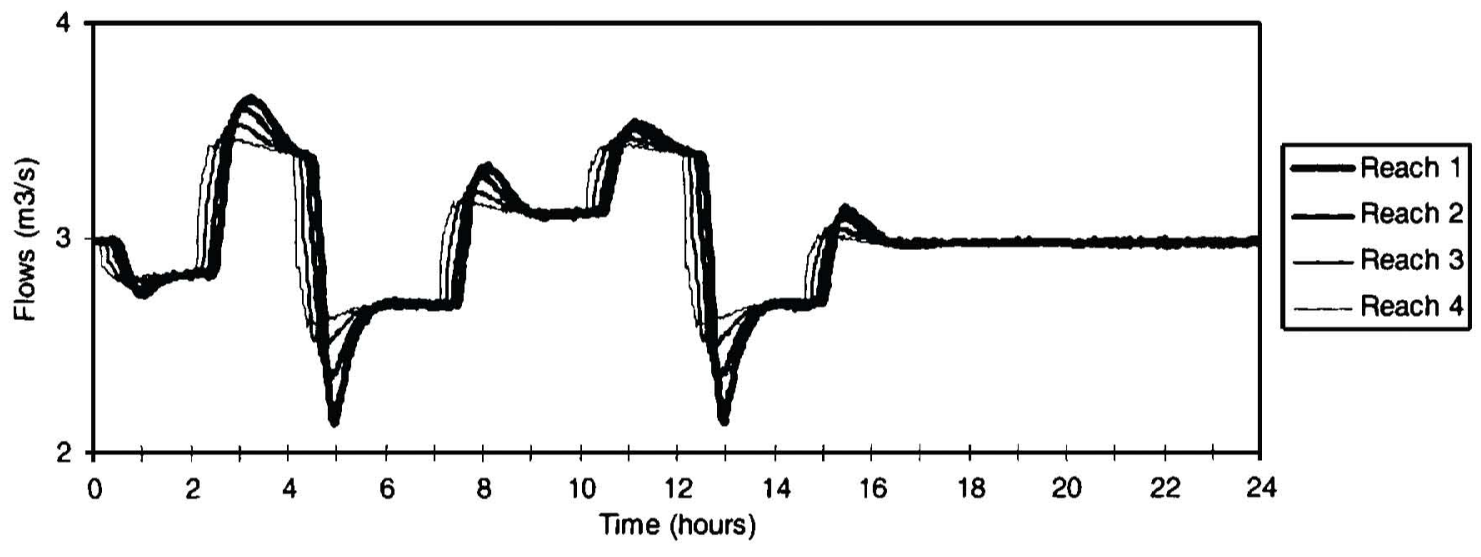

Fig. 6. Resulting flows 


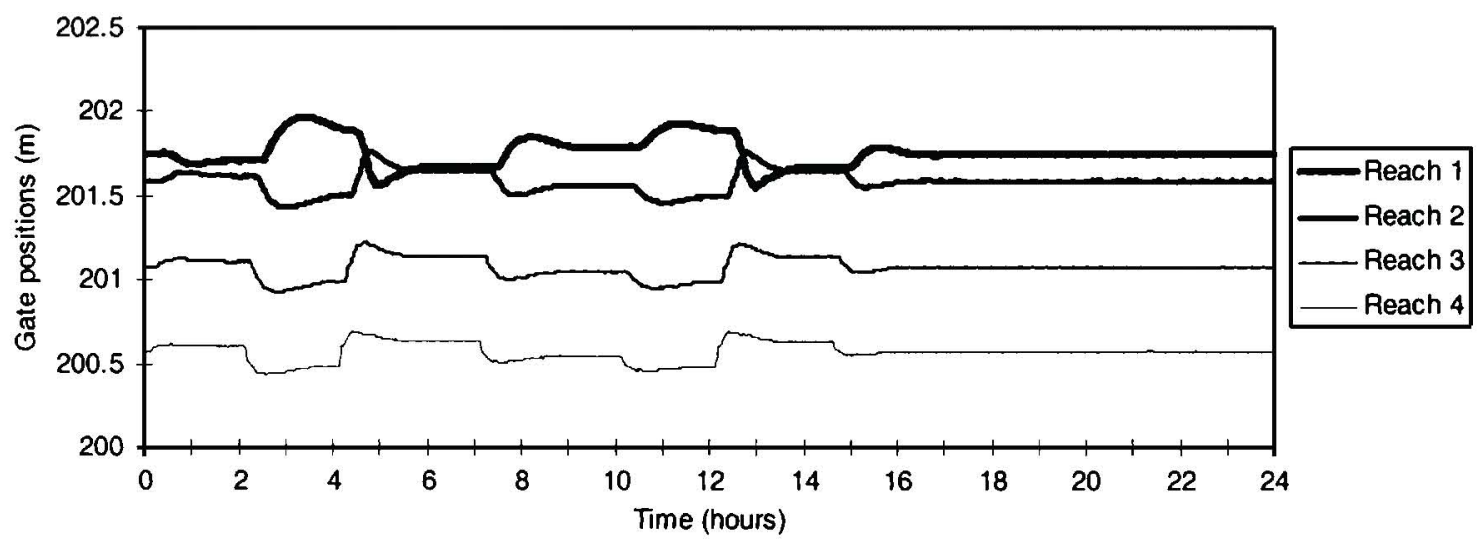

Fig. 7. Resulting gate positions

Mathematically, the objective function we use, $J$, can be described as

$$
J=\sum_{i=1}^{N} \lambda_{i} J_{i}
$$

where $\lambda_{i}=$ weight for the $i$ th model; $N=$ total number of models, and $J_{i}=$ objective function for the $i$ th model. The performance function $J_{i}$ is given by

$$
J_{i}=\sum_{k=0}^{\infty}\left[\mathbf{e}_{(k)}^{T} \mathbf{Q} e_{(k)}+\mathbf{u}_{(k)}^{T} \mathbf{R} u_{(k)}\right]
$$

where $\mathbf{e}_{(k)}=$ water-level deviation vector from target level at time $k$ in meters; $\mathbf{u}_{(k)}=$ control input vector at time $k$ in cubic meters per second; $\mathbf{Q}^{-}=$weighting matrix on water level deviation vector; and $\mathbf{R}=$ weighting matrix on control input vector.

The optimization problem consists of minimizing $J$ with respect to the control parameters that need to be tuned, subject to the $N$ models, where each model is perturbed by the same unit impulse disturbance.

For optimization, a method is needed for computing both the objective function and gradient of the objective function with respect to the variations in the control parameters. The value of the objective function could be found by simulating the response of each model to the disturbance, while the gradient could be computed numerically. However, for large canal networks such a method would require excessively long computation times and may encounter numerical problems. Fortunately, both the value and the gradient of the objective function can be calculated from the solution of a Lyapunov equation, which can be solved in a few iterations. A description of an earlier version of the optimization procedure is given in (Schuurmans and Liem 1995).

\section{Application of Multiple Model Optimization on Canals}

The multiple-model optimization procedure was programmed in Matlab (Mathworks 1992). Controllers tuned with this procedure have been applied to various accurate unsteady flow simulation models of irrigation canals. Examples include:

- El-Salam canal, Egypt, upstream PI control on 14 pools, modeled in SOBEK (WL/Delft Hydraulics 2000);

- Umatilla Stanfield Branch Furnish (USBF) Canal, Oregon, downstream PIF control on four pools, modeled in Sobek and CanalCAD (Holly and Parrish 1992); and

- Central California Irrigation District Canal, California, upstream PIF control on eight pools, modeled in CanalCAD.

The linear models that are used in the optimization are the integrator delay models at low and high flow, as these conditions

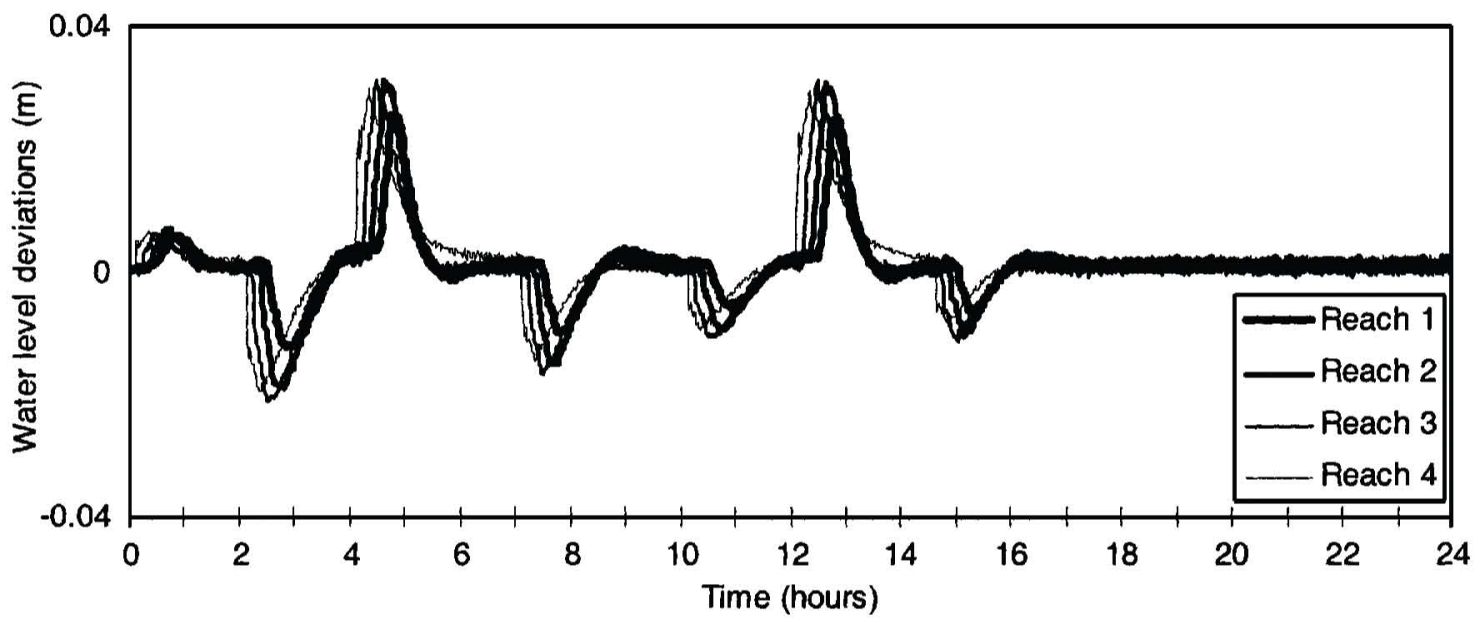

Fig. 8. Resulting downstream (controlled) water levels 
are the outer limits of all operating conditions. For decentralized control the delay times are negligible ( $\tau$ is set to zero), as there is hardly any delay between action and measurement. So the input requires the storage area $A_{s}$ of all pools at both low and high flow.

The control parameters need to be limited if significant resonance waves occur in the pools. In theory, resonance waves in a pool consist of an infinite number of harmonics. The frequency of the harmonics are determined by the dimensions of the pool. The higher harmonic that is in counterphase (phase lag is $\pi$ rad) with the control action can destabilize the control if the control gains are too high. More precisely, the gain margin must be smaller than 1. In the optimization procedure the upper limits on $K_{p}$ and $F_{c}$ are set according to Eqs. (3) and (5). Using the resonance peaks at low flow is the worst-case situation as these peaks are highest at low flow. The higher harmonic that is in counterphase with the control action is assumed to have its peak near the frequency of $\pi / T_{c}$. So the resonance peak $R_{p}$ at this critical frequency is an extra input parameter of the optimization procedure.

The pool characteristics that are required as input for the optimization can be determined by applying simple identification techniques (Silvis et al. 1998; Schuurmans 1997; Weyer 2001).

\section{Experimental Setup on the Umatilla Stanfield Branch Furnish Canal}

The potential of the multiple-model optimization is illustrated by an example of an existing canal. Fig. 2 shows the model of the Umatilla Stanfield Branch Furnish (USBF) Canal in Oregon. The canal consists of four pools in series divided by four structures (one undershot gate, three overshot gates). The total length of the canal is $8,600 \mathrm{~m}$. The four controlled pools are completely under backwater under all operating (flow) conditions. The water levels immediately downstream of the structures are controlled by the control structures with PIF control. Note that in this case $\tau$ is zero and $A_{s}$ is the surface area of the whole pool (see Fig. 1).

The canal and its structures were modeled in the hydrodynamic package SOBEK (WL/Delft Hydraulics 2000), while the controllers were programmed in Matlab (Mathworks 1992). In simulations these programs have an explicit coupling every control time step. In the tests a simulation time step of $1 \mathrm{~s}$ was used to make sure that all relevant dynamics (resonance waves) were included. The control time step was $1 \mathrm{~min}$. The maximum gate movement was limited to $120 \mathrm{~mm} / \mathrm{min}$, while the minimum gate movement was $3 \mathrm{~mm}$. This is according the actual implementation in the field.

The following tests were performed:

- Twelve-hour run with a $1 \%$ step change in the turnout flow in the last pool (all turnout flows in the other pools are zero). The controller is tuned with the initial controller settings. The controller parameters used are given in Table 1.

- Twelve-hour run with a $1 \%$ step change in the turnout flow in the last pool. The multiple-model optimization procedure was used to determine optimum tuning constants for the controllers. The tuned controller parameters are given in Table 2.

- Twenty-four hour run using a realistic turnout flow schedule in the last pool. In Fig. 3 the turnout schedule is shown. This is a typical turnout schedule over one day during the summer time. The optimized control parameters as given in Table 2 are used.

\section{Results}

Fig. 4 shows the result when the controllers on the USBF Canal are tuned with initial tuning rules for resonance-dominated pools that are designed to give a high performance in each pool. The disturbance amplification traveling from pool 4 to pool 1 can clearly be seen even on a small step change in flow of $1 \%$ at the downstream side of the canal.

Fig. 5 shows the result of the optimized decentralized PIF downstream control for the same $1 \%$ change in flow as used in Fig. 4. It is clear that tuning using a multiple-model optimization presents better control than received from tuning pools independently. The disturbance amplification evident in Fig. 4 has disappeared in Fig. 5.

Figs. 6-8 illustrate the simulated flows, gate positions, and water level deviations of the four pools when the realistic turnout schedule is used. It can be seen that the controlled water levels hardly fluctuate. An animation of the result can be seen at www.canalcontrol.com/usbfc.htm.

\section{Conclusions}

In the design of a centralized canal or open-water channel, decentralized control should be considered as an option, either as the main system or as the backup system. By applying a multiplemodel optimization that minimizes the water-level deviations from target level in all pools, the tuning of decentralized PI controllers on canals can be done in one design step, without an extensive trial-and-error procedure. This is irrespective of the number of pools that are lying in series. The optimization procedure presented in this paper can be used for upstream and downstream control. As the operating condition at low and high flow and the worst-case resonance peak are considered, the control is robustly stable under all operating conditions. The results show high performance (small water-evel deviations) and equally distributed maximum water-level deviations from target level (no disturbance amplification) in all pools.

\section{Notation}

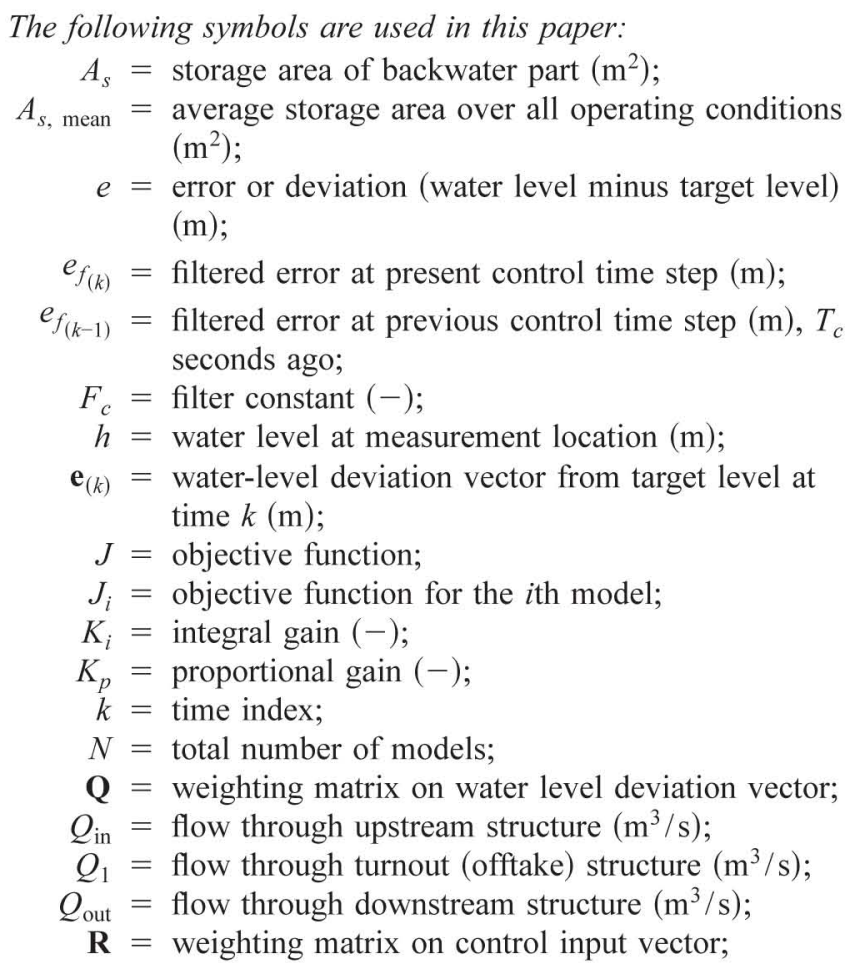




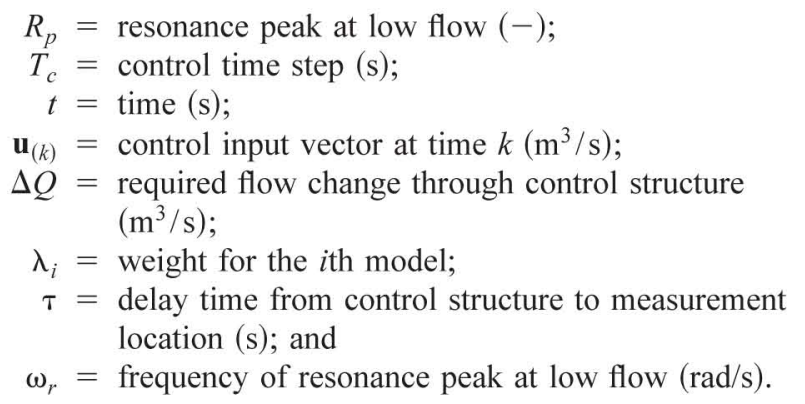

\section{References}

Geromel, J. C., and Bernussou, J. (1979). "Optimal decentralised control of dynamic systems." Automatica, 14, 489-491.

Holly, F. M., and Parrish, J. B. (1992). CanalCAD: Dynamic flow simulation in irrigation canals with automatic gate control, Iowa Institute of Hydraulic Research, Univ. of Iowa, Iowa City, Iowa.

Mathworks. (1992). Matlab user guide, Mathworks, Inc., Natick, Mass.

Overloop, P. J. van, Mursi Batt, A., Heeringen, K. J. van, and Thabet, R. A. H. A. (2001). "Real-time control of water quantity and water qual- ity in a reuse of drainage water project." Proc., International Commission on Irrigation and Drainage Workshop, South Korea.

Schuurmans, J. (1997). "Control of water levels in open channels." $\mathrm{PhD}$ thesis, Delft Univ. of Technology, Faculty Civil Engineering, Group of Water Management, The Netherlands.

Schuurmans, J., and Liem, G. (1995). "Controller design for the WM canal." Proc., Water Resources Engineering Conf., ASCE, New York, 481-485.

Schuurmans, W., et al. (1999). "Automatic control of Grand Prairie Project." Proc., United States Committee on Irrigation and Drainage Workshop, Phoenix.

Silvis, L. G., Hof, A., Hof, P. M. J. van den, and Clemmens, A. J. (1998). "System identification on open channels." Proc., 3rd Int. Conf. on Hydroinformatics, 219-224.

WL/Delft Hydraulics. SOBEK manual and technical reference, (2000). WL/Delft Hydraulics, Delft, The Netherlands.

Wahlin, B. T., and Clemmens, A. J. (2002). "Preliminary results for downstream feedback control of branching canal networks." Proc., United States Committee on Irrigation and Drainage/Environmental and Water Resources Institute Conf. on Energy, Climate, Environment, and Water, San Luis Obispo, Calif.

Weyer, E. (2001). "System identification of an open water channel." Control Eng. Pract., 9, 1289-1299. 